\title{
Blasenloser optoelektronischer Nivellierungssensor - theoretische Betrachtungen und Simulationen
}

\author{
R. Müller ${ }^{1}$, E. Förster ${ }^{2}$, O. Brodersen ${ }^{1}$ \\ ${ }^{1}$ CiS Forschungsinstitut für Mikrosensorik und Photovoltaik $\mathrm{GmbH}$, \\ Konrad-Zuse-Straße 14, 99099 Erfurt \\ rmueller@cismst.de \\ ${ }^{2}$ Fraunhofer-Institut für Angewandte Optik und Feinmechanik (IOF), \\ Albert-Einstein-Straße 7, 07745 Jena
}

\section{Kurzfassung:}

Auf Basis einer bekannten Strahler-Empfänger-Baugruppe wird der Aufbau eines optoelektronischen Nivellierungssensors vorgeschlagen. Die Messung erfolgt über ein Differenzverfahren und ist für zwei Neigungsachsen simultan möglich. Mittels Raytracing-Simulationen wurden Sensorkennlinien berechnet. Der Messbereich des Sensors liegt im Bereich von $\pm 0,3^{\circ}$.

Schlüsselwörter: optoelektronischer Neigungssensor, Strahler-Empfänger-Baugruppe, Strahlformung, Mikrooptik, Strahlenverfolgung.

\section{Einführung}

Für die Nivellierung von Analysenwaagen werden seit einigen Jahren sogenannte optoelektronische Libellensensoren eingesetzt (vgl. Abb. 1). Damit wird es möglich, den Anwender bei der manuellen Nivellierung der Waage zu unterstützen (Drehrichtung für $\mathrm{Füße}$ ) bzw. eine automatische Nivellierung der Waage durchzuführen. Ferner kann für jede Wägung der Nivellierungszustand der Waage dokumentiert werden.

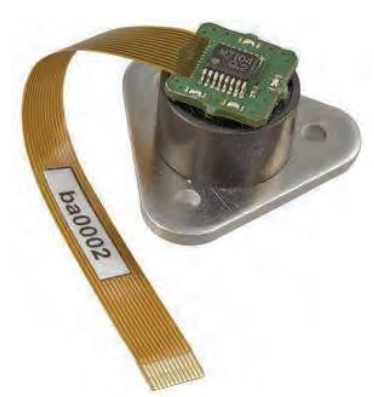

Abb. 1: Optoelektronischer Libellensensor.

Für bestimmte Anforderungen an Genauigkeit im Nullpunkt und Messbereich ergeben sich technische Probleme für die Realisierung von optoelektronischen Libellensensoren auf
Grund einer nichtlinearen Sensorkennlinie [1]. Hinzu kommt, dass die Dicke des Sensors maßgeblich von der Dicke der benötigten Dosenlibelle beeinflusst wird.

Insgesamt ist man an einem sogenannten blasenlosen optoelektronischen Nivellierungssensor interessiert (Verzicht auf Dosenlibelle).

\section{Funktionsprinzip}

Die Idee setzt auf einem am CiS Forschungsinstitut für Mikrosensorik und Photovoltaik $\mathrm{GmbH}$ bereits zur Verfügung stehendem System auf: Im Zentrum eines Siliziumsubstrates ist eine Kavität von $240 \mu \mathrm{m}$ Tiefe eingebracht. In dieser Kavität befindet sich ein vertikal emittierender Laser (VCSEL) mit einer Bauhöhe von $150 \mu \mathrm{m}$. Über dem Siliziumsubstrat ist ein Abstandshalter aus Foturan angebracht, der über dem VCSEL mit einem Durchbruch versehen ist (kreisrunde Bohrung). In diese Bohrung von oben hinein ragt eine so genannte Ringlinse. Diese formt das Licht des Emitters (VCSEL) zu einem Lichtring. Diese Ringlinse ist mit einem Träger aus Borofloat fest verbunden. In Abb. 2 ist eine beispielhafte Schnittdarstellung mit den wichtigsten Maßen für eine real existierende Anordnung dargestellt. 


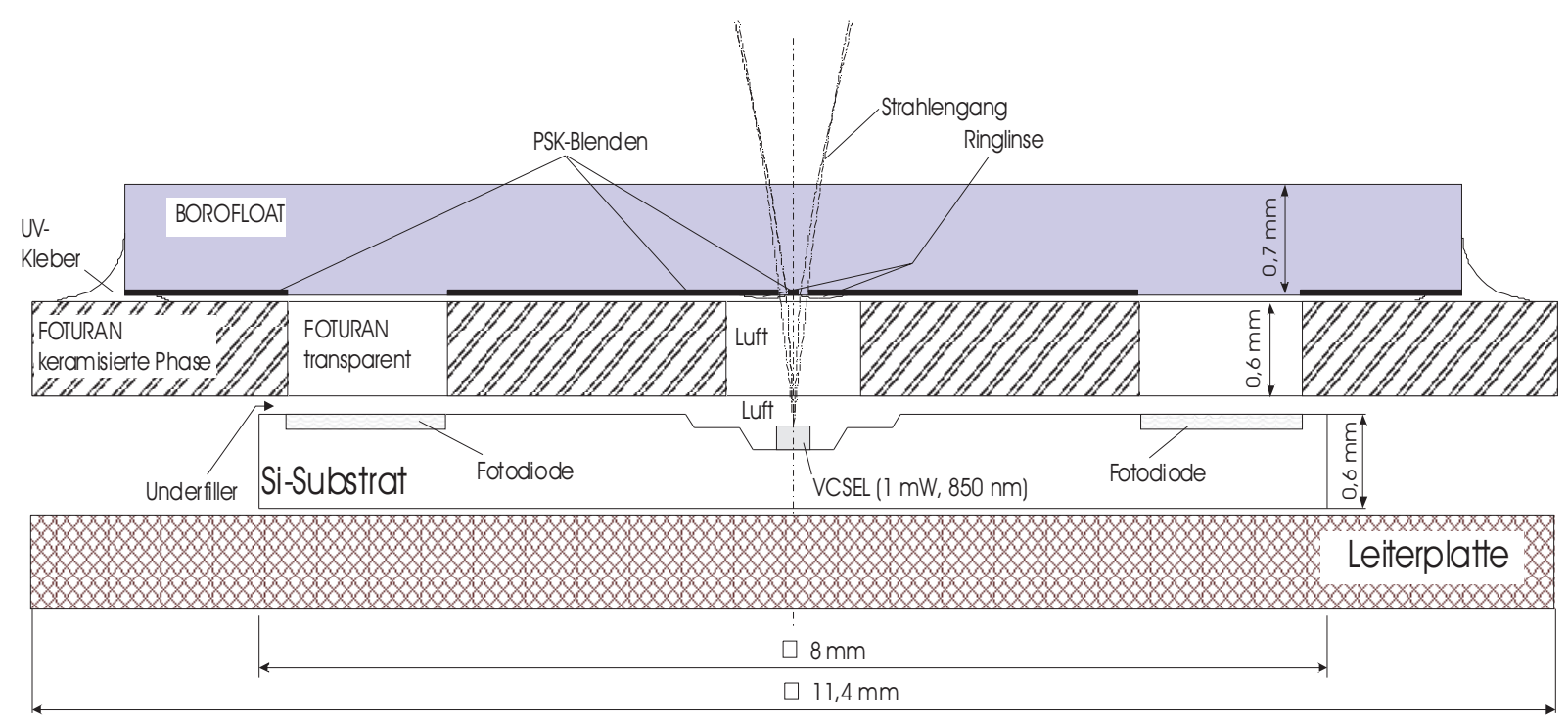

Abb. 2: Schnittdarstellung für eine am CiS entwickelte optoelektronische Strahler-Empfänger-Baugruppe mit integrierter Strahlformung.

In Abb. 3 ist die Lichtverteilung dargestellt, die sich bei Projektion auf einen Schirm gegenüber der Strahler-Empfänger-Baugruppe ergibt.

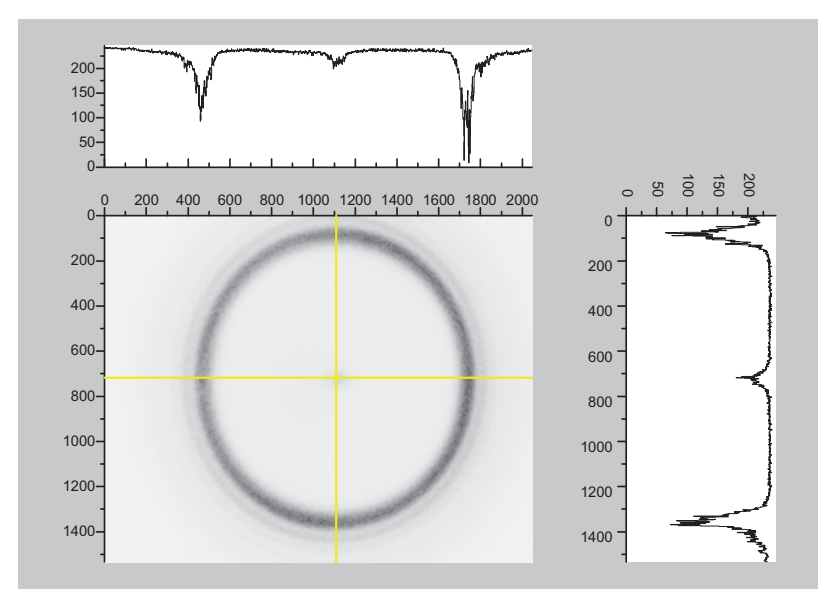

Abb. 3: Fotografische Darstellung der Lichtverteilung auf dem Schirm mit 8-bit Graustufen, der Abstand zwischen Laseraustrittsöffnung und PSKBlenden beträgt $670 \mu \mathrm{m}$, der Schirm ist $10,15 \mathrm{~mm}$ von der äußeren Borofloat-Oberfläche entfernt, die Bildfläche beträgt in X-Richtung $9,6 \mathrm{~mm}$ und in $Y$ Richtung 7,2 $\mathrm{mm}$.

Um die in Abb. 2 dargestellte Anordnung zu einem optoelektronischen Nivellierungssensor zu erweitern, befindet sich über dem Borofloat eine Schicht aus PMMA $(n=1,49)$, welche an der Oberseite mit einer Vertiefung in der Form eines Kegelstumpfes versehen ist. Über der PMMA-Schicht befindet sich eine Flüssigkeitsschicht, wobei die Flüssigkeit die Vertiefung im PMMA mit ausfüllt. Über der Flüssigkeit existiert eine Gasschicht. In Abb. 4 ist der vorgeschlagene rotationssymmetrische Aufbau im Schnitt mit den wichtigsten Maßen und dem Strahlengang dargestellt (Simulationsmodell).

Die Strahlung wird nach dem Übergang in die PMMA-Schicht zwei Mal total reflektiert. Die erste Totalreflexion findet an der Böschung des Kegelstumpfes statt (Grenzfläche: PMMA/ Flüssigkeit). Die zweite Totalreflexion findet an der Grenzfläche Flüssigkeit/Gas statt. Von hier gelangt die Strahlung auf die Fotodioden bzw. den absorbierenden Boden. Streuprozesse und Toleranzproblematiken werden hier vorerst nicht betrachtet.

Wird das System geneigt, verändert die Flüssigkeitsoberfläche ihre Lage zur Grenzfläche PMMA/Flüssigkeit. Es resultiert der Messeffekt - Die Fotoströme der beiden Detektoren kommen in einer gegenläufigen Wirkung aus dem Gleichgewicht. Als Sensorsignal kann z. B. die Differenz der beiden Fotoströme herangezogen werden. 

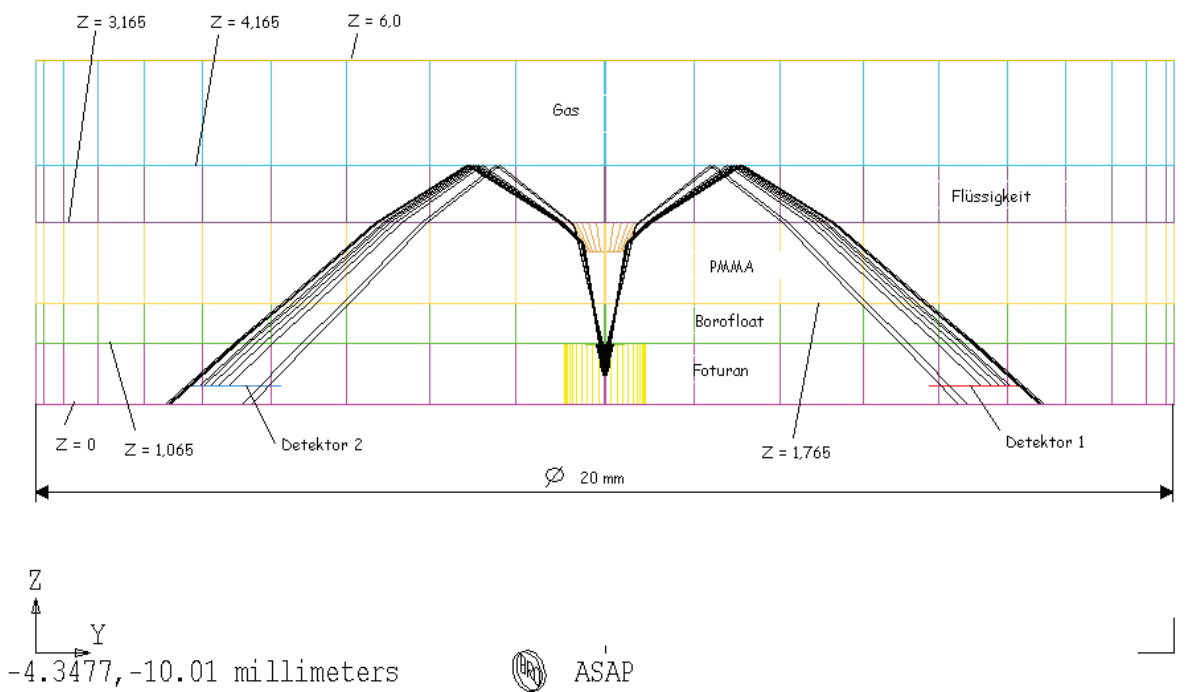

(969) ASAP

Abb. 4: Schnittdarstellung für das Simulationsmodell des optoelektronischen Nivellierungssensors mit Strahlengang (alle Maße in $\mathrm{mm}$ ).

\section{Ergebnisse der Simulationen}

Für die Brechzahl der Flüssigkeit wurde $n=1,333$ verwendet. Wie in Abb. 4 dargestellt kann somit die Lichtverteilung nach Abb. 3 bis in die Ebene der Fotodioden geführt werden. Für Kennlinienberechnungen für die Neigung um eine Achse wurden zwei Detektoren so ausgelegt (vgl. Abb. 5), dass der äußere Radius der Segmente in etwa dem Radius der maximalen Bestrählungsstärke entspricht $(7,25 \mathrm{~mm})$. Die Neigung der Flüssigkeitsoberfläche erfolgt gegen den Uhrzeigersinn. Die Drehachse verläuft in $X$-Richtung um den Punkt $X=0, Y=0, Z=4,165 \mathrm{~mm}$. Der Verlauf der optischen Leistung (1 $\mathrm{mW}$ Emission) auf den zwei Detektoren ist in Abb. 6 dargestellt.

Im austarierten Zustand empfängt jeder der beiden Detektoren eine optische Leistung von ca. $61,4 \mu \mathrm{W}$.

Dies entspricht bei einer Sensitivität von 0,6 A/W einem Fotostrom von 36,84 $\mu \mathrm{A}$. Im Bereich

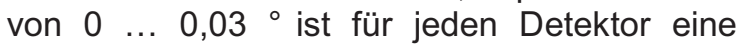
Änderung der empfangenen Leistung von ca. $1 \mu \mathrm{W}$ pro $0,01^{\circ}$ erreichbar.

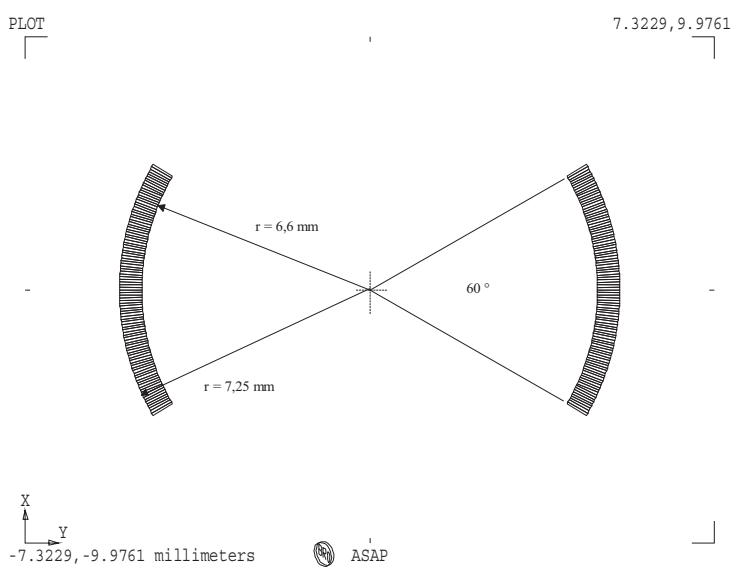

Abb. 5: Form der Detektoren.

Dieser Wert ist aber nicht überzubewerten, da keinerlei Toleranzbetrachtungen eingeflossen sind. Hier sind noch weitere Berechnungen notwendig. Prinzipiell wird als Sensorsignal die Differenz der Fotoströme von Detektor eins und Detektor zwei vorgeschlagen. Bei Einführung von zwei weiteren Detektoren, die um $90^{\circ}$ verdreht angeordnet sind, kann simultan die Neigung in zwei Achsen erfasst werden. 


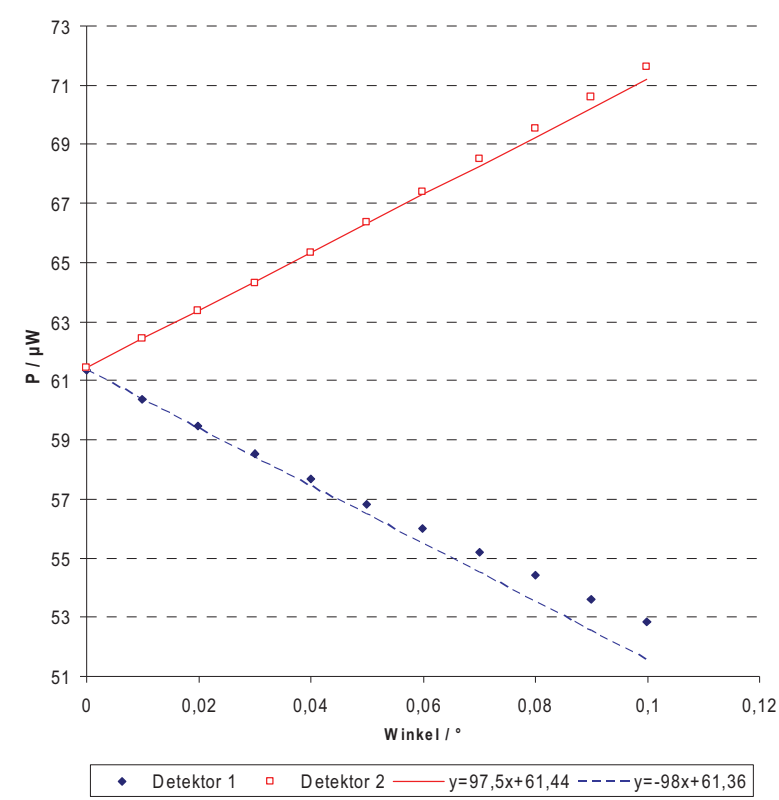

Abb. 6: Verlauf der optischen Leistung auf Detektor eins und zwei bei Verkippung der Flüssigkeitsoberfläche für den Aufbau nach Abb. 4.

Aus Abb. 6 ist weiterhin zu entnehmen, dass bis ca. $0,03^{\circ}$ eine betragsmäßig in etwa identische Steilheit für die beiden Detektoren berechnet wurde - dann weichen die Beträge der Steilheiten voneinander ab. Zusätzlich wurden zwei Geraden eingezeichnet, die durch die Verbindung der jeweils ersten beiden Kurvenpunkte gebildet wurden - dies visualisiert die oben gemachte Aussage.

In Abb. 7 ist der Verlauf der optischen Leistung auf den beiden Detektoren über einen größeren Winkelbereich dargestellt. Die Ursache für die unterschiedlichen Steilheiten liegt offenbar in der unsymmetrischen Verteilung der Bestrahlungsstärke im Profil des Lichtringes in der Ebene der Detektoren. Dies könnte durch gezielte Optimierungen der Strahlformung beseitigt werden.

\section{Schlussfolgerungen und Ausblick}

Ein kritischer Faktor für den Aufbau erster Funktionsmuster ist die Auswahl der Flüssigkeit. Eigenschaften wie Oberflächenspannung, Ausdehnungskoeffizient, Gefrierpunkt, Temperaturabhängigkeit der Brechzahl, Dampfdruck, Viskosität, Absorptionskoeffizient, Alterung usw. müssen berücksichtigt werden.

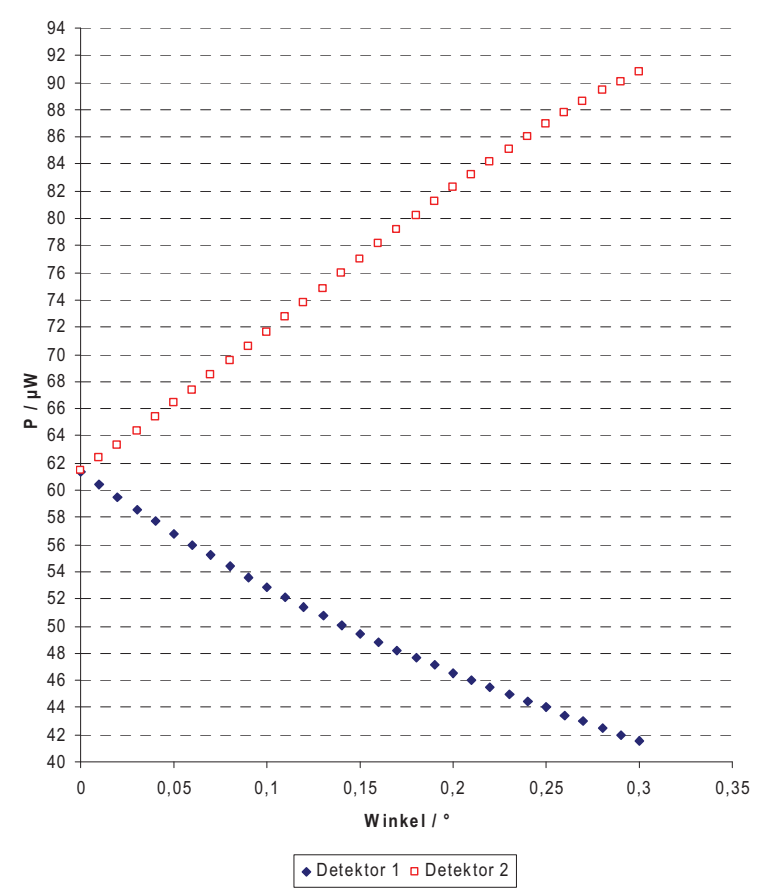

Abb. 7: Verlauf der optischen Leistung auf Detektor eins und zwei bei Verkippung der Flüssigkeitsoberfläche für den Aufbau nach Abb. 4 .

Für einen möglichst flachen Sensoraufbau ist eine dünne Flüssigkeitsschicht von Vorteil. Dies birgt aber die Gefahr einer unebenen Flüssigkeitsoberfläche bzw. einem schlechten Fließverhalten.

Zukünftig ist vorgesehen die PMMA-Schicht mit einer dünnen transparenten Platte abzudecken. So entsteht innerhalb des Kegelstumpfes ein Gasvolumen, welches die optischen Eigenschaften dieser Grenzfläche stabilisiert.

\section{Literatur}

[1] M. Zimmermann et al.: OPTO-ELECTRONICAL INCLINATION SENSOR. In: Proc. EUROSENSORS XXII, Dresden, 7.-10. September 2008, S. 253-256.

\section{Danksagung}

Die Arbeiten wurden unter dem Förderkennzeichen MF 110125 über die EuroNorm $\mathrm{GmbH}$ durch das Bundesministerium für Wirtschaft und Technologie innerhalb des Förderprogramms INNO-KOM-OST gefördert. Die Autoren danken für die Unterstützung. 